\title{
Fidelity Analysis for the OH-58D Kiowa Warrior Crew Trainer
}

\author{
John E. Stewart II \\ U.S. Army Research Institute \\ Kenneth D. Cross \\ Bayview Research \\ Robert H. Wright \\ U.S. Army Research Institute
}
Rotary-Wing Aviation Research Unit
Dennis C. Wightman, Chief

U.S. Army Research Institute for the Behavioral and Social Sciences 5001 Eisenhower Avenue, Alexandria, Virginia 22333-5600

June 1998 